УДК 517.547

\title{
O. B. SKASKIV
}

\section{PROGRESS IN THE OPEN PROBLEMS IN THEORY OF FUNCTIONS OF BOUNDED INDEX}

O. B. Skaskiv. Progress in the open problems in theory of functions of bounded index, Mat. Stud. 49 (2018), 109-112.

We give overview of solved problems in theory of functions of bounded index from the paper Bandura, A.I., Skaskiv, O.B.: Open problems for entire functions of bounded index in direction. Mat. Stud. 43(1), 103-109 (2015). Mostly problems concern with entire functions of several variables and analytic theory of differential equations. We pose also new problems.

We present overview of solved problems from [10] and give some remarks.

We need some notation and definitions. Let $L: \mathbb{C}^{n} \rightarrow \mathbb{R}_{+}$be any fixed continuous function. An entire function $F(z), z \in \mathbb{C}^{n}$, is called $([11,12])$ a function of bounded L-index in a direction $\mathbf{b}=\left(b_{1}, \ldots, b_{n}\right) \in \mathbb{C}^{n} \backslash\{\mathbf{0}\}$, if there exists $m_{0} \in \mathbb{Z}_{+}$such that for every $m \in \mathbb{Z}_{+}$ and every $z \in \mathbb{C}^{n}$

$$
\frac{1}{m ! L^{m}(z)}\left|\frac{\partial^{m} F(z)}{\partial \mathbf{b}^{m}}\right| \leq \max \left\{\frac{1}{k ! L^{k}(z)}\left|\frac{\partial^{k} F(z)}{\partial \mathbf{b}^{k}}\right|: 0 \leq k \leq m_{0}\right\},
$$

where $\frac{\partial^{0} F(z)}{\partial \mathbf{b}^{0}}:=F(z), \frac{\partial F(z)}{\partial \mathbf{b}}:=\sum_{j=1}^{n} \frac{\partial F(z)}{\partial z_{j}} b_{j}=\langle\operatorname{grad} F, \overline{\mathbf{b}}\rangle, \frac{\partial^{k} F(z)}{\partial \mathbf{b}^{k}}:=\frac{\partial}{\partial \mathbf{b}}\left(\frac{\partial^{k-1} F(z)}{\partial \mathbf{b}^{k-1}}\right), k \geq 2$.

For $\eta>0, z \in \mathbb{C}^{n}, \mathbf{b}=\left(b_{1}, \ldots, b_{n}\right) \in \mathbb{C}^{n} \backslash\{\mathbf{0}\}$ and a positive continuous function $L: \mathbb{C}^{n} \rightarrow \mathbb{R}_{+}$we define

$$
\lambda_{\mathbf{b}}(\eta)=\sup _{z \in \mathbb{C}^{n}} \sup _{t_{1}, t_{2} \in \mathbb{C}}\left\{\frac{L\left(z+t_{1} \mathbf{b}\right)}{L\left(z+t_{2} \mathbf{b}\right)}:\left|t_{1}-t_{2}\right| \leq \frac{\eta}{\min \left\{L\left(z+t_{1} \mathbf{b}\right), L\left(z+t_{2} \mathbf{b}\right)\right\}}\right\} .
$$

By $Q_{\mathrm{b}}^{n}$ we denote the class of functions $L$ such that $\lambda(\eta)$ is finite for any $\eta>0$. We also use the notation $Q=Q_{1}^{1}$.

Let us consider the following partial differential equation

$$
P_{1}\left(z_{1}, z_{2}\right) \frac{\partial F}{\partial z_{1}}+P_{2}\left(z_{1}, z_{2}\right) \frac{\partial F}{\partial z_{2}}=h\left(z_{1}, z_{2}\right)
$$

Problem 1 ([10, Problem 3]). Let $P_{1}\left(z_{1}, z_{2}\right), P_{2}\left(z_{1}, z_{2}\right)$ be entire functions of bounded $L$ index in directions $\mathbf{e}_{1}=(1,0)$ and $\mathbf{e}_{2}=(0,1)$ respectively. What are a direction $\mathbf{b}$ and additional assumptions such that every entire solution $F(z)$ of equation (1) has a bounded $L$-index in the direction $\mathbf{b}$ ?

2010 Mathematics Subject Classification: 30D15, 30D30, 34M05.

Keywords: bounded $L$-index in direction; entire function; several variables; bounded $\mathbf{L}$-index in joint variables. doi:10.15330/ms.49.1.109-112

(C) O. B. Skaskiv, 2018 
A. A. Goldberg observed [13] that the following algebraic partial differential equation

$$
z_{1} \frac{\partial F}{\partial z_{1}}-z_{2} \frac{\partial F}{\partial z_{2}}=0
$$

is satisfied by the function $w=f\left(z_{1} z_{2}\right)$, where $f(u)$ is an arbitrary entire function. But there are known sufficient conditions of $L$-index boundedness for composition of entire functions [9]. In view of these results, we conclude that if function $f(u)$ has bounded index then the function $f\left(z_{1} z_{2}\right)$ has bounded $L_{\mathbf{b}}$-index in the direction $\mathbf{b}=\left(b_{1}, b_{2}\right) \in \mathbb{C}^{2} \backslash\{0\}$ with $L_{\mathbf{b}}\left(z_{1}, z_{2}\right)=$ $\left|b_{1} z_{2}+b_{2} z_{1}\right|+1$. In other words, we need some additional assumptions (for example, initial conditions) to certainly describe behavior of entire solutions of (1). Of course, this is also true for more general equation [10, Problem 1]

$$
f_{1}(z) \frac{\partial F}{\partial \mathbf{b}_{1}}+f_{2}(z) \frac{\partial F}{\partial \mathbf{b}_{2}}=h(z) .
$$

The similar conclusion is valid for the following problem.

Problem 2 ([10, Problem 2]). Let $g\left(z_{1}, z_{2}\right)$ be an entire function of bounded $L$-index in the directions $\mathbf{b}_{1}$ and $\mathbf{b}_{2}$. What are a function $L^{*}$ and a direction $\mathbf{b}^{*}$ that an entire solution of equation $\frac{\partial^{2} F}{\partial \mathbf{b}_{1} \partial \mathbf{b}_{2}}=g\left(z_{1}, z_{2}\right)$ has a bounded $L^{*}$-index in the direction $\mathbf{b}^{*}$ ?

Indeed, for $\mathbf{b}_{1}=(1,0), \mathbf{b}_{2}=(0,1)$ and $g\left(z_{1}, z_{2}\right) \equiv 0$ we have that any entire function $F\left(z_{1}, z_{2}\right)=f\left(z_{1}\right)+g\left(z_{2}\right)$ is a solution of $\frac{\partial^{2} F}{\partial z_{1} \partial z_{2}}=0$, where $f$ and $g$ are arbitrary entire functions. Thus, if we choose entire functions with unbounded multiplicities of zeros as $f$ and $g$ and such that $f(0)=0$ and $g(0)=0$ then the function $F\left(z_{1}, z_{2}\right)$ is of unbounded $L$-index in the direction $(1,0)$ and $(0,1)$ for any positive continuous function $L: \mathbb{C}^{n} \rightarrow \mathbb{R}_{+}$. This means that the problem also requires initial conditions to certainly describe behavior of entire solutions.

Remind Problem 4 from [10]. Let us consider the ordinary differential equation

$$
w^{\prime}=f(z, w) \text {. }
$$

Problem 3 ([10, Problem 4]). Let $f(z, w)$ be a function of bounded L-index in directions $\mathbf{e}_{1}=(1,0)$ and $\mathbf{e}_{2}=(0,1)$. What is a function $l$ such that an entire solution $w=w(z)$ of equation (2) has a bounded l-index?

The problem is completely solved in [8]. Moreover, there was considered more general equation

$$
w^{(p)}=f(z, w) .
$$

We denote $\mathbf{e}_{1}=(1,0), \mathbf{e}_{2}=(0,1)$. Let us write

$$
G_{r}(F):=G_{r}^{\mathbf{b}}(F)=\bigcup_{z: F(z)=0}\{z+t \mathbf{b}:|t|<r / L(z)\},
$$

and let $a_{k}^{0}$ be zeros of the function $F\left(z^{0}+t \mathbf{b}\right)$ for a given $z^{0} \in \mathbb{C}^{n}$. By $n_{z^{0}}(r, F)=$ $n_{\mathbf{b}}\left(r, z^{0}, 1 / F\right):=\sum_{\left|a_{k}^{0}\right| \leq r} 1$ we denote the counting function of the zeros $a_{k}^{0}$ of the slice function $F\left(z^{0}+t \mathbf{b}\right)$ in the disc $\{t \in \mathbb{C}:|t| \leq r\}$. If for a given $z^{0} \in \mathbb{C}^{n}$ and for all $t \in \mathbb{C}$ $F\left(z^{0}+t \mathbf{b}\right) \equiv 0$, then we put $n_{z^{0}}(r)=-1$. Denote $n(r)=\sup _{z \in \mathbb{C}^{n}} n_{z}(r / L(z))$. Our solution is provided by the following statement.

Proposition 1. Let $l_{j} \in Q_{\mathbf{e}_{j}}^{2}$, and $f(z, w)$ be an entire function of bounded $l_{j}$-index in the directions $\mathbf{e}_{j}$ for every $j \in\{1,2\}$. If there exist $C>0$ and $l \in Q$ such that for all $(z, w) \in \mathbb{C}^{2} \backslash\left(G_{r}^{\mathbf{e}_{1}}(f) \cup G_{r}^{\mathbf{e}_{2}}(f)\right)$

$$
l_{1}(z, w)+l_{2}(z, w)|f(z, w)| \leq C l(z),
$$


then every entire function satisfying (3) has bounded l-index.

Besides, we conjectured possibility of replacing universal quantifier by existential quantifier (Conjectures 1 and 2 in [10]) in some criteria of $L$-index boundedness in direction.

Problem 4 ([10, Problem 6]). Is Conjecture 1 true?

Conjecture 1. Let $L \in Q_{\mathbf{b}}^{n}$. An entire function $F(z)$ is of bounded $L$-index in direction $\mathbf{b} \in \mathbb{C}^{n}$ if and only if there exist $R>0, P_{2}(R) \geq 1$ and $\eta(R) \in(0, R)$ such that for all $z^{0} \in \mathbb{C}^{n}$ and some $r=r\left(z^{0}\right) \in[\eta(R), R]$ inequality $\max \left\{\left|F\left(z^{0}+t \mathbf{b}\right)\right|:|t|=\frac{r}{L\left(z^{0}\right)}\right\} \leq$ $P_{2} \min \left\{\left|F\left(z^{0}+t \mathbf{b}\right)\right|:|t|=\frac{r}{L\left(z^{0}\right)}\right\}$ holds.

Conjecture 1 is completely proved in $[7,11]$.

Problem 5 ([10, Problem 7]). Is Conjecture 2 true?

Conjecture 2. Let $F(z)$ be an entire in $\mathbb{C}^{n}$ function, $L \in Q_{\mathbf{b}}^{n}$ and $\mathbb{C}^{n} \backslash G_{r}^{\mathbf{b}}(F) \neq \varnothing$. $F(z)$ is a function of bounded $L$-index in the direction $\mathbf{b} \in \mathbb{C}^{n}$ if and only if: 1) there exist $r>0$, $P=P(r)>0$ such that for each $z \in \mathbb{C}^{n} \backslash G_{r}^{\mathbf{b}}(F)$ inequality $\left|\frac{1}{F(z)} \frac{\partial F(z)}{\partial \mathbf{b}}\right| \leq P L(z)$ holds;

2) there exist $r>0, \widetilde{n}(r) \in \mathbb{Z}_{+}$such that for every $z^{0} \in \mathbb{C}^{n}$, for which $F\left(z^{0}+t \mathbf{b}\right) \not \equiv 0$, and for all $t_{0} \in \mathbb{C}$ inequality $n_{\mathbf{b}}\left(\frac{r}{L\left(z^{0}\right)}, z^{0}, \frac{1}{F}\right) \leq \widetilde{n}(r)$ holds.

Conjecture 2 is partially proved in $[7,11]$. Particularly, we proved the following statement.

Theorem. Let $L \in Q_{\mathrm{b}}^{n}, F(z)$ be an entire function in $\mathbb{C}^{n}$. If the following conditions hold: 1) there exist $r_{1}>0, \quad P>0$ such that for each $z \in \mathbb{C}^{n} \backslash G_{r_{1}}(F)$ inequality $\left|\frac{1}{F(z)} \frac{\partial F(z)}{\partial \mathbf{b}}\right| \leq P L(z)$ holds; 2) there exists $r_{2}>0$ such that $n\left(r_{2}\right) \in[-1 ; \infty)$ and $2 r_{1} \cdot n\left(r_{2}\right)<r_{2} / \lambda_{\mathbf{b}}\left(r_{2}\right)$, where $r_{1}$ is chosen from previous condition, then the function $F$ is of bounded L-index in the direction $\mathbf{b}$.

Note that Conjecture 2 from [10] is proved under the additional restriction $2 n\left(r_{2}\right) r_{1}<$ $r_{2} / \lambda_{\mathbf{b}}\left(r_{2}\right)$. It is currently unknown whether the condition is essential.

Problem 6 ([11, Problem 3]). Denote $r_{0}=\sup _{r>0} \frac{r}{2 n(r) \lambda(r)}$. Are there an entire function $F$ in $\mathbb{C}^{n}$ and a continuous function $L: \mathbb{C}^{n} \rightarrow \mathbb{R}_{+}$with properties: 1) there exist $r_{1}>r_{0}$ and $P>0$ such that for each $\left.z \in \mathbb{C}^{n} \backslash G_{r_{1}}^{\mathbf{b}}(F) \frac{1}{|F(z)|}\left|\frac{\partial F(z)}{\partial \mathbf{b}}\right| \leq P L(z) ; 2\right)$ for any $r_{2}<r_{0}$ $\sup _{z \in \mathbb{C}^{n} \backslash G_{r_{2}}^{\mathbf{b}}(F)} \frac{1}{|F(z)| L(z)}\left|\frac{\partial F(z)}{\partial \mathbf{b}}\right|=+\infty$ and $n\left(r_{2}\right) \in(0 ;+\infty) ?$

Also there was formulated the following problem.

Problem 7 ([10, Problem 17]). What are conditions on the zero set and growth of entire functions providing the index boundedness of $F\left(z_{1}^{0}+b_{1} t, z_{2}^{0}+b_{2} t\right)$ for every $\left(z_{1}^{0}, z_{2}^{0}\right) \in \mathbb{C}^{2}$ and the index unboundedness of $F\left(z_{1}, z_{2}\right)$ in the direction $\mathbf{b}=\left(b_{1}, b_{2}\right)$ ?

We find some answer to the question in [6]. There was proved that if $f(t), t \in \mathbb{C}$, is an even entire transcendental function of bounded index then 1) for each direction $\mathbf{b}=$ $\left(b_{1}, b_{2}\right) \in \mathbb{C}^{2} \backslash\{0\}$ and for every fixed $z_{1}^{0}, z_{2}^{0} \in \mathbb{C}$; the function $g(t)=f\left(\sqrt{\left(z_{1}^{0}+b_{1} t\right)\left(z_{2}^{0}+b_{2} t\right)}\right)$ is an entire function of bounded index $(t \in \mathbb{C})$; 2$) f\left(\sqrt{z_{1} z_{2}}\right)$ is of unbounded index in each direction $\mathbf{b}$. 
Another interesting property of entire function o bounded index has discovered by W. K. Hayman([14]). An entire function $f(z)$ is said to have bounded value distribution (Turán, 1953) if there exists constant $p, R$ such that equation $f(z)=\omega$ never has more than $p$ roots in any disc of radius $R$. W. K. Hayman([14]) proved that every entire function is a function of bounded value distribution if and only if its derivative is a function of bunded index.

In view of this property, we pose the following problem.

Problem 8. What are operators preserving index boundedness for analytic functions?

Conjecture 3. Differential operators preserving univalence also must preserve index boundedness for some additional assumptions.

\section{REFERENCES}

1. Bandura A., Skaskiv O., Entire functions of bounded $\mathbf{L}$-index: Its zeros and behavior of partial logarithmic derivatives, J. Complex Analysis, 2017 (2017), 1-10.

2. Bandura A.I., Skaskiv O.B., Analytic functions in the unit ball of bounded L-index: asymptotic and local properties, Mat. Stud., 48 (2017) №1, 37-73.

3. Bandura A., Skaskiv O., Asymptotic estimates of entire functions of bounded $\mathbf{L}$-index in joint variables Novi Sad J. Math., 48 (2018) №1, 103-116.

4. Bandura A.I., Skaskiv O.B., Growth of entire functions of bounded L-index in direction, Mat. Met. Phys.-mech. fields, 60 (2017) №1, 22-31.

5. Bandura A.I., Skaskiv O.B., Sufficient sets for boundedness L-index in direction for entire functions, Mat. Stud., 30 (2008), №2, 177-182.

6. Bandura A.I., A class of entire functions of unbounded index in each direction, Mat. Stud., 44 (2015), №1, 107-112.

7. Bandura A.I., Some improvements of criteria of L-index boundedness in direction, Mat. Stud., 47 (2017), №1, 27-32.

8. Bandura A., Skaskiv O., Filevych P., Properties of entire solutions of some linear PDE's, J. Appl. Math. Comput. Mech., 16 (2017), №2, 17-28. doi: 10.17512/jamcm.2017.2.02

9. Bandura A., Composition of entire functions and bounded L-index in direction, Mat. Stud., 47 (2017), №2, 179-184. doi: 10.15330/ms.47.2.179-184

10. Bandura A.I., Skaskiv O.B., Open problems for entire functions of bounded index in direction, Mat. Stud., 43 (2015) №1, 103-109.

11. Bandura A.I., Skaskiv O.B., Directional logarithmic derivative and the distribution of zeros of an entire function of bounded L-index along the direction, Ukrain. Mat. J., 69 (2017), №3, 500-508.

12. Bandura A., Skaskiv O., Entire functions of several variables of bounded index, Lviv: Publisher I.E. Chyzhykov, 2016, 128 p.

13. Goldberg A.A., On the growth of entire solutions of algebraic differential equations, Lithuanian Mathematical Journal, 45 (2005) №1, 44-49.

14. Hayman W.K., Differential inequalities and local valency, Pacific J. Math., 44 (1973) №1, 117-137.

Ivan Franko National University of Lviv

Lviv, Ukraine

olskask@gmail.com 